\title{
Moral Intuitions vs. Moral Reasoning. A Philosophical Analysis of the Explanatory Models Intuitionism Relies On
}

\author{
Sara Dellantonio and Remo Job
}

\begin{abstract}
The notion of 'intuition' is usually contrasted with rational thought, thus motivating a differentiation between two kinds of processes that are supposed to characterize human thinking, i.e. rational and 'intuitive' (immediate and non-argumentative) forms of judgment. Recently, the notion of intuition has also played a leading role in cognitive studies on morality with the rise of so-called social intuitionism, according to which people's moral stances are culturally driven intuitions - i.e. they are quick, involuntary and automatic responses driven by culturally and socially acquired principles (see e.g. 42], 41] and [22]). Usually, intuitionism is presented as radically opposed to rationalistic views of morality according to which moral judgments are the outcome of explicit reasoning. In this work we compare two different hypotheses concerning the possible relationship between reasoning and intuition: a 'continuist interpretation' (maintaining that intuitions and judgments based on reasoning are produced by the same cognitive process) and a 'discontinuist interpretation' (supporting the view that they are produced by two different cognitive processes). We argue that a continuist interpretation appears more plausible than a discontinuist one and that the concepts of 'intuition' and 'reasoning' are two facets of the same process which spans from fast, immediate, and certain answer to slow, conscious and elaborate judgments. According to this interpretation, moral judgments are produced by the same kinds of inferences reasoning relies on, i.e. mostly deduction, induction and abduction. Our analysis will show that to opt for a continuist interpretation has many consequences for the way morality is explained from a psychological point of view. Mainly, it challenges the idea of morality
\end{abstract}

Sara Dellantonio · Remo Job

Dipartimento di Scienze della Cognizione e della Formazione,

Universitá degli Studi di Trento

e-mail: sara.dellantonio@unitn.it, remo.job@unitn.it

L. Magnani and P. Li (Eds.): Philosophy and Cognitive Science, SAPERE 2, pp. 239 262 springerlink.com

(C) Springer-Verlag Berlin Heidelberg 2012 
proposed by intuitionism, according to which moral intuitions are rigidly driven by culturally learned principles.

Our reflections lead rather to the conclusion that the first and spontaneous intuitions fully enculturated people may experience do not often express the best moral judgment possible in a certain situation, but are rather the product of the prejudices people inherit from their culture/subculture. This gives rise to the conclusion that people are better guaranteed to form truly moral judgments when they do not respond intuitively to morally relevant situations, but interrupt and override this automatic processing, moving on to a controlled i.e. a rational process.

\section{Introduction}

The notion of 'intuition' has continued to be influential in the philosophical tradition since the pre-Socratics. Over time, however, it has evolved taking on deeply different connotations. In contemporary philosophical studies, intuition is viewed as an immediate, simple, passive, non-verbal procedure of knowledge acquisition (see e. g. 43]). In cognitive science, this notion is usually contrasted with rational thought, thus motivating a differentiation between two kinds of processes that are supposed to characterize human thinking, i.e. rational and 'intuitive' (i.e. immediate and non-argumentative) forms of judgment 1 . Recently, intuition has also played a leading role in cognitive studies on morality and moral sense, since it is considered an ideal concept to describe the way in which people produce their moral judgments.

Within this dualistic view of moral judgment, it has been proposed that people's moral stances are culturally driven intuitions - i.e. that they consist of quick, involuntary and automatic responses driven by culturally and socially acquired principles (see e.g. 42, 41, and 22]) - and that these intuitions are the product of an innately programmed moral module in the brain (see e.g. 28, and 30]). More precisely, intuitions are defined as "the sudden appearance in consciousness of a moral judgment, including an affective valence (good-bad, like-dislike), without any conscious awareness of having gone through steps of searching, weighing evidence, or inferring conclusions. [...] One sees or hears about a social event and one instantly feels approval or disapproval." (22 p. 818). At least according to Haidt's social form of intuitionism, the good/bad evaluations produced by moral intuitions "are made with respect to a set of virtues held to be obligatory by the culture or the subculture." (22] p. 817 )

Usually, intuitionism is presented as radically opposed to the previous rationalistic view of morality (see e.g. 22, 23 and 24) supported by a large part of the classical philosophical studies and by the psychological tradition that starts with Piaget and continues with Kohlberg and Turiel (see e.g. [47, [35] and [55]), according to which moral judgments are the outcome of explicit

\footnotetext{
${ }^{1}$ For an overview see e.g. 9].
} 
reasoning. Such reasoning is considered to be a form of conscious reflection or of verbalized deliberation that proceeds slowly and with effort, weighing up motives and principles.

Intuitionism tries to account for the fact that in order to form a moral judgment people often do not reason about an issue or weigh up the different aspects of a situation. Rather, their answer regarding the right thing to do seems to come up immediately and spontaneously, and its content tends to conform to the rules and the habits of the culture or group they belong to. Indeed, this same evidence constitutes the starting point of many contemporary cognitive theories about morality (like e.g. the Rawlsian and Humean ones: see [4). In this sense, intuitionism is surely right in saying that, to be plausible, moral theories need to explain why moral judgments appear (at least mostly) to be intuitive rather than reflective. Still, we think that the concept of 'intuition' which intuitionism appeals to hasn't been defined precisely enough from the point of view of the cognitive processes that are supposed to produce intuitions. The following questions need at least to be investigated: What kind of process gives rise to intuitions? In what respect does this cognitive process differ from the one that it is supposed to produce reasoning?

In this work we compare two different hypotheses concerning the possible relationship between reasoning and intuition. I.) On the one hand, we consider the hypothesis that our intuitions (i.e. the fast and immediate answers people produce in certain cases, without having doubts or being aware of the reasons supporting them) and reasoning (the slow, reflective and often beset by doubts form of thought people sometimes perform) are produced by the same cognitive process, using the same kind of information. We will call this a 'continuist interpretation' of the relationship between rationalism and intuitionism. II) On the other hand, we will consider the idea that intuitions and judgments based on reasoning are produced by two different kinds of cognitive processes and therefore really do differ cognitively from each other, as assumed by intuitionists. We will call this a 'discontinuist interpretation' of the relationship between rationalism and intuitionism. On the basis of this comparison we will argue that a continuist interpretation appears more plausible than a discontinuist one and that the concepts of 'intuition' and of 'reasoning' do not cognitively differ, i.e. they do not refer to the outputs of two different cognitive processes, but are two facets of the same process which spans from fast, immediate and certain answer and to slow, conscious and elaborate judgments. According to this interpretation, moral judgments are produced by the same kinds of inferences reasoning relies on, i.e. mostly deduction, induction and abduction.

This thesis concerns only moral judgments. In this sense it is not a claim against dual theories or the massive modularity view outright, according to which the mind works using two radically different systems or processing mechanisms: a modular system, which works rapidly and automatically and 
a non modular system that produces complex, hypothetical and decontextualized thought, which is flexible and able to reach high abstraction levels (see e.g. [10, 48, 7] and [53]). However, as far as moral cognition is concerned, the hypothesis we put forward here is surely incompatible with dual-systemtheories, but still compatible with more recent dual-processing-theories that differentiate between various "types" or "levels" of processing, leaving open the possibility that they might be generated by the same system ([8] and [16]).

Our analysis will show that to opt for a continuist interpretation has many consequences in terms of the way morality is explained from a psychological point of view. Mainly, it challenges the idea of morality proposed by intuitionism, according to which moral intuitions are rigidly driven by culturally learned principles, and to be morally virtuous simply means to be "fully enculturated", i.e. to have assimilated the moral principles of a culture or subculture and to follow them slavishly. Our reflections lead rather to the idea that the first and spontaneous intuitions fully enculturated people may experience do not often express the best moral judgment possible in a certain situation, but are rather the product of the prejudices people inherit from their culture (or subculture). A parallelism with socio-psychological studies on this aspect is proposed. This gives rise to the conclusion that people are better guaranteed to express truly moral judgments when they do not respond intuitively to morally relevant situations, but rather interrupt and override this automatic processing, moving on to a controlled i.e. a rational process.

\section{Intuitions and Inferential Reasoning According to a Continuist Interpretation}

Apparently, the distinction between moral reasoning and moral intuition is clear and sharp, even easy to observe in our everyday experience. And apparently intuitionism is right in maintaining that our 'moral' judgments seem to be, at least in the large majority of cases, entirely 'intuitive', since they are fast and since they are not beset by doubts, while in just a few particularly difficult situations, involving different and possibly contradictory aspects (like the moral dilemmas made up in the laboratory using artificial scenarios) people have recourse to a slow and reflective form of reasoning in order to form moral judgments. Still, the fact that our moral judgments are experienced as being mostly intuitive and that intuitions are experienced as cognitive processes which radically differ from reasoning does not guarantee that there is an actual difference in the nature of the cognitive process that produces what we perceive to be an intuition and what we perceive to be a form of reasoning. In fact, in this respect questions arise about what kind of cognitive process may produce moral intuitions and whether this process is really qualitatively different from the one underlying reasoning. 
To follow up this line of investigation we need firstly to consider whether it is possible to explain intuitions and judgments based on reasoning as two apparently different products of the same cognitive process. We will call this a 'continuist interpretation' of the relationship between intuitions and judgments based on reasoning.

Intuitions and judgments based on reasoning are usually considered to differ from each other first of all because intuitions are produced without people being conscious of the possible reasons supporting the judgments they intuitively formed. However, the unconscious nature of the cognitive processes that lead to an output is not a distinctive feature of moral intuitions. In fact, the idea that the way we form our thoughts (the information we use and the steps we follow) is in general for the most part not accessible to consciousness is one of the essential tenets of the cognitive sciences, which states that cognition consists in information processing, of which only the final product is accessible to consciousness. According to a classic computational view, thought is produced by a central system that processes information on the basis of logical and inferential relations that refer to the semantic properties of the information processed. As Fodor makes clear "[...] the notion of computation is intrinsically connected to such semantic concepts as implication, confirmation, and logical consequence. Specifically, a computation is a transformation of representations which respects these sorts of semantic relations." (13 p. 5) According to this view, when I hear for instance a sentence like 'Cleo is lying on the floor', my immediate understanding of it and my automatic reaction to her - I run towards Cleo - will depend on information processing which will follow more or less a path such as:

Cleo is a fish $\Rightarrow$ Outside the water fishes die $\Rightarrow$ On the floor there isn't any water $\Rightarrow$ Either Cleo has already died or she will soon, unless I immediately put her back in the water.

Even though this information processing is the condition for understanding the sentence I hear and its consequences, I don't need to be conscious of the path it followed to understand the sentence and to react to it. In fact, people are generally not conscious that they are processing information in this way and they may not even be able to reconstruct the information process through which they came to understand a sentence when they are requested to explain it. Even if they are able to do so, it will cost them a lot of effort to make explicit and verbalize linguistically the inferential path that produced the understanding. And in any case the explanation of the inferential path is just a post hoc reconstruction; and one can never be sure that the reconstruction corresponds to the actual inferential path that has taken place. As for the understanding of the sentence itself it will appear to the subject as an unconscious, immediate, spontaneous and non-reflective intuition.

What this example shows is that we can reach a specific conclusion (the understanding of something, but also a certain judgment) on the basis of inferential processing on semantically structured information without being 
conscious that this information is being processed. This processing may also start spontaneously, be fast and not require any particular reflection. But, if so, then both our intuitive (fast, spontaneous, unconscious) answers and our reasoned (slow, conscious) judgments could be the results of information processing which relies on logical and inferential operations on semantically structured information. This semantically structured information could be that which concepts are composed of (see also [4]).

Indeed, according to mainstream research on semantics (contra Fodor's atomism: see e.g. [14], [15]) concepts are composed of different pieces of information: According to this view, to know what a fish is - i.e. to have the concept of 'fish' - means for example to know (at least) that fishes are animals, that they can live only in water, that they have round open eyes, fins, and commonly a typical rounded-stretched form, that they don't have legs, etc; the concept of 'fish' must therefore be made up of these 'pieces of information' (in the literature on concepts they are more often called 'features 2 ). These features do not codify perceptual information only, i.e. information about the external aspect of the conceptualized things; they also codify the common, widely shared and well-grounded knowledge people have about objects: someone who knows e.g. what a fish is (i.e. who has the concept of fish), generally knows a lot of things about fishes such as: they are mostly edible, some of them are considered pets, they eat insects, lay eggs, etc. Thus, this information is also part of the concept of fish.

To claim that all kinds of judgments are the results of information processing amounts to stating that all kinds of judgments - intuitive or reasoned - are the results of information processing which relies on logical and inferential operations on concepts and on these concepts' features. A general idea about how this might work can be given using the example above: since the concept of 'fish' includes the information 'animal' and 'animal' includes the information 'mortal', one can infer that - being an animal - a fish is mortal. In general, the semantically structured pieces of information that compose concepts can be connected with each other to form chains of deductive and inductive inferences as well as other more complicated forms of inferences that we use in our reasoning processes like abductive inferences, which are indispensable for forming hypotheses.

Smith states that: "An intimate relation connects inductive inferences and categorization; namely, categorizing an object licenses inductive inferences about that object. For example, if we see a round, reddish object on a tree and categorize it as an apple, we can then infer that is edible and has seeds. Thus categorization is the mental means we have for inferring invisible properties from visible ones." ([50] p.6) Even though Smith focuses primarily on inductive inferences, the categorization process as he describes it also involves deduction and abduction. If I see a round, reddish object on a tree and all the round, reddish objects I have seen on trees in the past turned out to be

\footnotetext{
${ }^{2}$ For a technical overview of the featural approach see e.g. 50, pp. 10-22.
} 
apples, I can inductively infer that this round, reddish object is an apple too. But, if I know that all apples are edible and have seeds, then I can deductively infer that, if this is an apple, it will be edible and have seeds. As far as abduction is concerned 3 this is used to form hypothesis about the objects we have categorized (about their behavior and their connections with others). Referring to the example, abduction is used in cases like:

Cleo is on the floor $\Rightarrow$ therefore, she has jumped out of her bowl.

What happens from a cognitive point of view, when we make this inference is something like:

\begin{abstract}
observation: Cleo (my goldfish) is on the floor $\Rightarrow$ surprising effect: Cleo should not be on the floor, she should be in her bowl, where I left her $\Rightarrow$ elaboration of an hypothesis to explain the event: (fishes can jump) if Cleo had jumped out of her bowl, this would explain why she is now on the floor; (given what I know about fishes and about other circumstances regarding the environment where the bowl was located) no other hypothesis can explain the event as well as this one $\Rightarrow$ conclusion: hence, Cleo must have jumped out of her bowl.
\end{abstract}

What this example suggests is first of all that there is an intimate relation between concepts and inferential reasoning in all its forms; more precisely: that all forms of thought based on concepts work on logical and inferential operations. In this sense, all forms of thought based on concepts are in a way rational, if by rational we simply mean 'based on logical and inferential operations' applied to available information'.

This conclusion allows us to now specify more precisely the position expressed by a 'continuist interpretation' of the relationship between reasoning and intuitions: according to a continuist interpretation, both (fast and automatic) intuitions and (conscious) reasoning are forms of thought based on concepts - i.e. they are products of logical and inferential operations on concepts and on the pieces of information concepts consist of; for this reason and in this respect they are both in a sense rational.

According to this interpretation, the word 'rationality' does not describe conscious forms of information processing only; nor does this notion of rationality include any guarantee that the conclusion of an inference will be 'rational' in the sense of 'the best possible' all things considered. In fact, the logical and inferential operations on concepts we are speaking about are based just on the specific pieces of information a person has acquired about the world and is able to include in a specific occurrence of information processing and this information is often very limited, inaccurate, reciprocally incoherent, and most importantly oversimplified and affected by prejudices. (We will say more about this later.)

Furthermore, the idea that all forms of thought are inferential information processing based on logical and inferential operations (i.e. that they are in

\footnotetext{
${ }^{3}$ For a classical definition of abduction see e.g. Peirce [44] $\S 188-189$; for a contemporary discussion on abductive inferences see e.g. [58] ch. 1; [38] ch. 1, 2 .
} 
a way rational) is not in conflict with the possibility that emotions play a part in processing information. Even the simplified example of the sentence 'Cleo is lying on the floor' shows on the contrary that emotions are always part of information processing, since the chain of thoughts that follow the understanding of this sentence as well as our practical reaction to it are both highly dependent on whether and how much we care that Cleo may be dying. So, it is obvious that information isn't 'emotionally neutral' for a human information processing system, which manifestly exhibits a lot of complex 'positive and negative propensities' towards specific things (it cares/doesn't care for specific things, it likes/doesn't like, it fears/desires etc. certain others). In fact, this particular (positive or negative) 'emotional attachment' to specific pieces of information characterizes all forms of thought (judgments, opinions, decisions). It is the particular form of this attachment that drives e.g. our reaction to the sentence 'Cleo is lying on the floor': a positive attachment to Cleo makes us run to save her, while a negative one makes us wait a little longer. This applies to all forms of thought/opinion/conclusion from the simplest ones such as 'The bus is leaving in five minutes' (Do we care? How much do we care? Is it worth running and e.g. giving up our morning coffee?) to the most socially complex ones like 'In some places children starve to death' (Do we care? How much do we care? Is it worth giving up some of our income to help them?). In this sense we can consider all kinds of inferential processes as driven not only by logical and inferential relations among pieces of information but also by the specific emotional connotations of specific pieces of information.

This description of the inferential processes at the basis of our thinking raises a question: why couldn't moral intuitions just be a form of thought, i.e. why couldn't they be the conclusion of a inferential process like the one that leads to the understanding of and appropriate reaction to the sentence 'Cleo is lying on the floor'? And, if we rely on a weak notion of rationality, why couldn't intuitions and judgments based on reasoning both be realized by the same procedure of the kind just described? In order to support such a continuist interpretation of intuition and reasoning, we need to explain why people experience a difference between these two kinds of process, the one being fast, immediate, sure and unconscious, and the other being slow, reflective and beset by doubts, even though both were produced by the same mechanism.

One possible answer to this is that intuitions and judgments based on reasoning are experienced as different cognitive modalities because they are the expression of two possible courses of the supposed inferential information processing. When the information process proceeds without 'hitches' i.e. when the logical and inferential operations on the available information go on without running into a contradiction or obstacle of some kind and do not encounter any novel or surprising situation that needs to be weighed up carefully - their results appear to our consciousness in the form of quick, immediate, and spontaneous intuitions. In contrast, when the information 
process encounters an impasse, (which may also be caused by confrontations with other people and/or by the need to find explicit arguments in support of a position), then it takes the form of reasoning - i.e. a reflective, conscious, slow and difficult form of thinking. A soldier may, for example, come intuitively to the conclusion that it is morally permissible to torture his/her prisoner to draw information out of him/her. Still, if he also happens to conceive of his/her prisoner as a person fighting for what he/she believes in and for his/her people, the soldier's moral position may reach an impasse which needs to be solved. In sum, finding a way to restore the coherence of the system is a necessary condition in order to arrive at a judgment about the moral legitimacy of torturing the prisoner.

So, to sum up, according to a continuist interpretation, intuitions and judgments based on reasoning could be produced by the same inferential process, while the difference the subject experiences between them could be due to the fact that inferential processes may take different paths: when the process does not encounter any obstacles, subjects experience the conclusion as an intuition, when, on the other hand, the process does meet an obstacle, coherence needs to be restored, the process slows down, different possibilities are explored, and sometimes new information is collected. In this case the subject experiences the conclusion of the process as reasoned.

\section{The Phenomenon of 'Dissidence'}

The explanation given in the previous section opens the door to the possibility of a continuist interpretation of intuitions and judgments based on reasoning, according to which they are produced by the same cognitive mechanism which sometimes proceeds without 'hitches' and appears to be fast, immediate, sure and unconscious, while at other times encounters an impasse or runs into a contradiction of some kind or encounters a novel or surprising situation and takes the form of a conscious, slow and difficult form of thinking. However, the possibility of arguing for a continuist interpretation of intuitions and judgments based on reasoning does not ipso facto exclude that a 'discontinuist interpretation' is also plausible and that authors like Haidt who favor an account based on intuitions are right in maintaining that intuitions are radically different from reasoning. According to such an interpretation, intuitions are not produced by the same cognitive process as reasoning, rather they are produced by an automatic and less flexible (modular) mechanism than reasoning which works just with a specific type of information, i.e. with moral information.

Haidt maintains that this mechanism is set by the moral principles of the cultural group or subgroup people are part of, while moral intuitions are directly triggered by the mechanism itself (see e.g. [22] and [28). So, drawing some examples from Haidt, if a culture sets the individuals' mechanism according to the principles 'incest is always forbidden' or 'abortion is always 
forbidden', people belonging to that culture will always have the intuition that abortion and incest are morally forbidden in any case. This intuition will be immediate, unequivocal and unquestionable and will come up in the form of a strong feeling of right or wrong. In fact, in consequence of this interpretation, Haidt states that moral virtue and full cultural integration are one and the same thing: "a fully enculturated person is a virtuous person" (24] p. 216). So, once the modular mechanism is set up by the culture, its working will be strongly bound to the principles it works with and leave very little space for change and flexibility.

A discontinuist interpretation clearly more closely adheres to the intuitionists' point of view since it accounts for the idea that moral reasoning and moral intuitions are not only experienced as different kinds of judgments, but they actually are different kinds of judgment since they are produced by two different processing systems. While reasoning is produced by a flexible system that can make use of all kinds of information, carry out all kinds of inferences, reflect and draw conclusions, intuitions are rigidly driven by culturally learned principles. The point of intuitionism is that, when we form or express a moral position, we do so not on the basis of reasoning, but rather on the basis of intuition. Still, this interpretation runs into difficult problems. A first and fundamental one is that it gives rise to a concept of morality that does not correspond to what have always been considered truly moral attitudes and stances.

In fact, morality cannot consist of a supine allegiance to the norms and customs of a group. As both the classic philosophical and psychological tradition have shown, morality cannot merely consist in blindly following a rule, without evaluating whether this rule is morally right or not (see e.g. 33] for the philosophical tradition and [35] for the psychological one). On the contrary, truly moral forms of thought and behavior are those which are capable of breaking away from the norms and the customs of a particular group in order to follow different principles, which are considered as right independently of what it is stated by the group or sub-group one belongs to. This behavior has indeed been placed by Kohlberg at the $5^{\circ}$ and higher level of his moral developmental scale (see e.g. 35) and is supposed to be based on the individual's capacity to critically and autonomously evaluate the right moral behavior in a given situation. From this perspective, one of the moral conditions par excellence is the phenomenon of dissidence, i.e. a form of disagreement expressed at a certain point by a member of a group about a principle, or about a position belonging to the common ideological framework of the group (see also 4]). Among the most well known examples of dissidence is the case of Nazism and of those German Aryanists that adhered, at least at the beginning, to National Socialism, but later helped Jews to save themselves, betraying in so doing the ideals of their group and infringing the racial law in force.

The fact that this phenomenon poses a problem for social intuitionism has already been pointed out very clearly by Nervaez, who uses Kohlberg's 
position to make a critical point against Haidt and Bjorklund: "In the early years of the moral developmental tradition, there was a distinction made between social conformity and moral development (35). The distinction was necessary in order to explain how in some situations (e.g. Germany in the 1930s) social conformity worked against moral development, and in others resisting social pressure (U.S. civil rights movement of the 1950s and 1960s) was a virtuous path. Thus, it is shocking to read Haidt and Bjorklund assert that 'a fully enculturated person is a virtuous person' (24 p. 216). Apparently Hitler youth and Pol Pot's Khmer Rouge were virtuous and most moral exemplars are not." (40 p. 239) And further: "[...] how does social intuitionist theory judge the goodness or badness of particular intuitions? Intuitions appear to be equally meritorious, as are all cultural practices, if they conform with the norms of one's social group ('full enculturation'). This is precisely the attitude that drove Kohlberg to mount his research program - how to support the law-breaking behavior of Martin Luther King, Jr., and condemn the law-abiding behavior of the Nazi soldier." (40] p. 240)

Nervaez's objection applies to all views that, like social intuitionism, consider morality as the output of an automatic cognitive process, which is uniform for all people of the same group and which is driven by the moral principles sanctioned by that group. The problem with such views is that they do not account for the autonomy of moral positions with respect to the moral principles accepted and shared by a group or sub-group. A theory of moral cognition may take the position that dissidence isn't an emblematic expression of moral behavior. Still, since the phenomenon of dissidence constantly occurs in history and since it has always been considered as a genuine moral stance both by the people who took a dissident position and by the people witnessing the situation from a point of view external to the group, it needs to be accounted for by a theory of moral cognition aiming to provide a comprehensive explanation of the human 'moral sense'. The phenomenon of dissidence suggests that humans have the capacity to morally act in a way that infringes the moral principles and conventions embraced by the community, group or sub-group they belong to. This means that people do not merely follow the moral principles embraced by their group, but they are also able to identify, work out and weigh up critically and autonomously moral principles and moral behaviors. For this reason they may arrive at a judgment that diverges from, or is opposed to, the one expressed by the norms of the customs of their group.

According to theories that assume moral judgments are intuitions driven by social principles, all kinds of traceable differences among moral intuitions can only be ascribed to cultural differences, or more precisely to more or less fine-drawn differences among the principles people happened to learn during their life. There isn't any reason in principle to exclude that the phenomenon of dissidence can be explained in this same vein as the consequence of the fact that different people are 'exposed' to different cultural information or have 'assimilated' different cultural elements. Still, in order for this proposal to 
hold, intuitionist theories need to clarify why and how this may happen: i.e. what does this different 'exposure' and 'assimilation' concretely consist of and which kinds of information among the varieties available are relevant to direct subjective moral intuitions in one direction or in another. The problem here, of course, is that, since each of us belongs concurrently to different groups and subgroups, allowing very subtle idiosyncratic cultural differences to affect our moral judgment, we must admit that each of us is determined by his/her own unique cultural experience. But, in this case, the notion of "enculturation" would become explanatorily useless.

In addition, such an explanation will be difficult to sustain on a theoretical level if we adopt a discontinuist interpretation of intuitionism. According to such an interpretation intuitions aren't the product of the central system, but of a module, i.e. by definition a mechanism which is much more inflexible and informationally encapsulated than the central system and which can hardly rearrange itself and become sensitive to new information. Such a mechanism must therefore be almost insensible to new information acquired by the system after the time when it is first set (encapsulation). This mechanism must also be quite resistant against distortions (i.e. untouched in its modus operandi) brought about by new information. These characteristics make it particularly difficult to explain cases like the phenomenon of dissidence in which moral judgment changes radically over time. For such a change to happen the modular mechanism for the production of moral judgments must be both quite permeable to new information (even to information which is opposed to specific culturally dominant moral principles) and quite flexible in order to turn the old operational mode into a new one and find a new assessment after assimilating new information. If we take perception as an emblematic example of cognition produced by modular mechanisms - as is usually done, and as Haidt does as well (see e.g. 22 p. 814 ) - we can easily face the problem with flexibility and encapsulation of modules: the way we perceive neither changes over time when we acquire new information, nor is it influenced by information other than that specific information needed to first set up the mechanism and which the mechanism has access to.

Haidt admits that inflexibility and encapsulation pose a problem for a theory of moral cognition and maintains that intuitionism needs for this reason to rely on a weaker idea of modularity like the one proposed by the so called 'massive modularity hypothesis' (p.es. [27, [28). Still, even if we give up completely or to a large extent the idea that modules have the properties of being rigid and encapsulated (at the risk, by the way, of making the modularity thesis lose its sense, since the supposed modules could become identical to the central system), intuitionism - i.e. the thesis of morality as full enculturation - does not allow us to explain why someone can became a dissident even though he/she is and continues to be part of a group/sub-group that upholds different moral values. In other words, interpreted according to a discontinuist interpretation, intuitionism cannot explain why the moral 
judgment of a person can change without any correspondent modification in the cultural environment he/she is exposed to.

\section{The Slow Processing of Morality}

Why and how might a person change his/her moral judgment over time, infringing the cultural principles he/she first learned? In the previous section we tried to show that a continuist interpretation of intuitionism and reasoning provides us with better theoretical means to answer this question, because it suggests that, if necessary - i.e. when the situation is perceived as novel and or presents obstacles, impasses or contradictions - people may modify the way they produce their moral judgments. They can shift from intuitions to slow and conscious reasoning, adding and weighing more and more elements in their inferential processing. According to a continuist approach, the inferential apparatus deployed in producing moral judgments is the same whether a fast or a slow response is provided.

Both the continuist and discontinuist interpretations are compatible with the idea that the fast and intuitive way of processing a moral output is cognitively realized using what in the literature on rationality and decision making are called "heuristics", i.e. specific procedures that speed up thinking processes allowing a parsimonious search for information and giving rise to immediate and spontaneous answers that are perceived as intuitions 4 However, the idea of what a heuristic is and above all what effects the application of a heuristic has on the processes that lead to the production of a moral judgment radically differ in the two cases.

Because heuristics ignore potentially relevant information, they have always been considered error-prone and less-than-optimal procedures (see e.g. 31 and 32]). Still, recent studies have shown that heuristics may be adaptive and ecologically useful (see e.g. [19] and 20]) hence suggesting that they may be, at least in some cases, preferable to reasoning, including moral reasoning. Indeed, some authors have implicitly or explicitly maintained that there is nothing wrong with the use of heuristics to produce moral judgments. This has been implicitly assumed by Haidt when he states that moral evaluations are (and can only be) intuitions driven by cultural values (see e.g. 22. and 24), and it has been explicitly put forward by Gigerenzer, in discussing Haidt's position (18, pp. 18ff). While it might be the case that for decision making intuitions-as-heuristics often provide positives outcomes, for moral

\footnotetext{
${ }^{4}$ Some authors explicitly connect the idea that moral judgments might be produced on the basis of heuristics with social intutionism; it is e.g. Gigerenzer who says: "[...] moral intuitions as described in the social intuitionist theory (e.g. 22 ) can be explicated in terms of fast and frugal heuristics" (18 p. 9; see also 17]). However, this idea is equally or possibly even more compatible with a continuist approach, according to which heuristics are just a procedure applied by the central system to speed up its processes.
} 
dilemmas they may be misleading since they might not be the result of some cultural moral principle but, rather, of some cultural prejudice.

A case that may help in clarifying the risk of applying intuitions when we are requested to evaluate a person or a situation from a moral point of view is that of 'stereotypes' as they are defined by social psychology as a form of heuristic used by people to speed up their judgments about a social situation. Social psychologists define 'stereotypes' as "knowledge structures" that people use to categorize groups or specific members of groups ([52] pp. 1-8). Such knowledge structures tend to work on the basis of a limited number of attributes and to disregard individual differences, leading to unwarranted generalizations about individuals and groups. Just like heuristics, stereotypes simplify information processing (see e.g. 11, 12, 36, 37 and 34) by reducing variability in the input and by tracing something newly experienced back to already available knowledge structures. Furthermore, as in the case of heuristics, the activation of stereotypes occurs quickly, automatically, spontaneously and effortlessly, without intention or consciousness, when we first categorize a person as a member of the group we have stereotyped (see e.g. 2 and [57]).

Stereotypes have a strong cultural base, and are powerful tools for processing in-group/out-group relationships. For this reason, they tend to associate positive properties with the members of one's own group(s) and negative properties with the members of other groups. It is such cultural filtering of information that make stereotypes special cases of intuitions: They have played and may still play an adaptive role by preserving in-group safety, but they cannot be taken as morally positive stances since individuals are judged not on the basis of what they are, but on the basis of what the prejudices about the group they belong to suggests they may be.

For instance, we could intuitively judge that it is morally legitimate to restrain gypsies from moving freely from country to country because they might rob other people's properties. But in so doing we are ascribing to each individual gypsy the feature 'thief' that may apply to some of the group members. Conversely, we may suspect a gypsy of robbing something as the result of the prejudice that - since he is a gypsy - he must surely be the person responsible for robbery (if not presently, at least in some other cases) and therefore he deserves to be punished. Thus, independently from any consideration regarding whether the use of heuristics as short cuts in replacement for longer reasoning processes have to be considered adaptive or ecologically useful as regards the reaction or interaction they elicit toward the categorized instances, from a moral point of view they cannot be viewed as optimal. And their sub-optimality, or plain wrongness, is due to the fact that they are culturally filtered, i.e. they are the product of people's 'full enculturation', and therefore of the cultural prejudices people may have. From this point of view, while intuitions may still give rise to correct moral judgment, the very fact that they rely on limited conceptual information and, as in the case of 
stereotypes, culturally filtered information, makes them less reliable as far as the output of the moral judgment is concerned.

According to the discontinuist interpretation, the moral positions people express are produced by an intuitive, modular system specialized for the processing of moral outputs and separated from the central system that carries out reasoning processes. According to this view, the reasoning process starts only after the moral module has produced its output and therefore cannot influence or intervene in the work of the moral module or change its output, but only deliver a post-hoc justification of the output produced by the module, whatever this output may be. In this approach the question about what is morally right or wrong can only be decided on the basis of intuitions and never on the basis of reasoning. So, from this approach it follows that the intuitions we form on the basis of our prejudices are the only moral positions we are able to produce: people don't have an alternative to making moral judgments on the basis of their own prejudices. In the continuist as contrasted with the discontinuist interpretation, this view on moral judgment changes completely because it is no longer assumed that the production of moral judgment is the exclusive prerogative of the intuitive system. In fact, by hypothesizing that intuitions and reasoning are not produced by two separate, independent systems, but rather by a single process that can proceed either quickly, using a limited amount of information, or slowly including more information, we conclude that both what we call reasoning and what we call intuitions can produce moral judgments.

Besides being highly undesirable, the conclusion of the discontinuist approach is also not plausible because we are de facto at least potentially capable of escaping our own prejudices and producing moral evaluations based on complex information, even when stereotypes are available. In fact, the example of stereotypes allows us to point out that a person can voluntarily generate obstacles when trying to escape his/her own prejudices. As some results obtained in the field of social psychology show, if people are motivated to challenge their own prejudices, reasoning might contribute to identifying them, to discovering that they are at work, and to reducing their effect on a final judgment 5 Such a possibility was pointed out by Allport as early as

\footnotetext{
${ }^{5}$ Social psychological models make various hypotheses about the relation between intuitions produced immediately and spontaneously on the basis of our prejudices and reasoning, which can be activated consciously to overcome these prejudices. Some models tend to stress the duality of judgment processes, suggesting that, when someone weighs up a situation or a person, he/she can give either an intuitive evaluation or a reasoned judgment, (see e.g. [45]), while other models assume that the two type of processes occur in parallel and can affect each other (see e.g. [51]). We suggest that in reality both situations can occur and can be descriptively appropriate, since we sometime evaluate a situation only intuitively or only on the basis of a reasoning process, while at other times we spontaneously give an intuitive response to a situation, while concurrently activating a reasoning process about it.
} 
1954 in The Nature of Prejudice, where he maintains that people can "put the brackets on their prejudices" ([1] p. 332). More recently the possibility of escaping prejudice has been considered more diffusely (e.g. [1] and [5]). In particular, it has been shown that people are able to intentionally inhibit stereotypes, and the influence of these stereotypes on judgments, and to replace them with other kinds of knowledge on the matter ([5]). Further, that this control cannot be engaged without becoming aware of the presence of a prejudice: i.e. without a conscious reflection concerning the fact that a bias is at play (see e.g. [3], 6]). The possibility pointed out by social psychology of escaping from our first prejudicial intuition turns out to be compatible with a continuist interpretation. According to this view, we usually produce our moral evaluations using fast and frugal heuristics, but when we have grounds to avoid short cuts and easy solutions, we are able to slow down the process and to reason using a larger amount of information.

\section{Morality, Culture and Educational Level}

Haidt's orthodox discontinuist interpretation suggests that the truly moral responses are intuitions, whose content is entirely determined by the cultural principles and values peoples have assimilated. As a consequence of this view people of the same cultural group share a large and strong intersubjective agreement with respect to their moral positions, while the contents of the moral positions of different groups may differ greatly from each other. However, as we will try to show here, this idea of a wide in-group moral uniformity, accompanied by a wide trans-group moral dissimilarity conflicts with some phenomena pointed out by moral psychology with relation to human moral responses. In particular, we will present some data driven by moral literature that, taken together, indirectly support our view, at least since they show that an increase in people's information-i.e. an increase in the features that qualify the concepts they rely on in their reasoning processes 6 - changes people's moral stances, decreasing the influence of their culture and forming something like a trans-group similarity among moral judgments.

\footnotetext{
${ }^{6}$ The notion of 'information' is used here in a purely cognitive sense to mean the features our concepts and conceptions are made of. This notion has nothing in common with that of 'information systems' or of 'information made available by the media', since - in opposition with some philosophical views like Habermas' - we don't think that an increase of the information made available in a society by the media would lead to a more moral and democratic system (see e.g. 21]). Cognitive capacities of humans are indeed very limited and are usually focused on specific tasks or aspects. An increase in the general information available could just be ignored, or not be correctly assimilated or lead to confusion and misunderstandings. On the contrary, when we appeal to a form of reasoning that applies to the information our concepts and conceptions are made of, we are speaking of information already available to the subjects, whose features can become explicit for him/her.
} 
The best way to introduce this point is in terms of the contraposition of Turiel's and Heid's views on morality. Throughout the course of his research Turiel has been trying to show that human beings are equipped with the basic capacity to distinguish moral violations from merely conventional violations. According to Turiel's definition: "Conventions are part of constitutive systems and are shared behaviors (uniformities, rules) whose meanings are defined by the constituted system in which they are embedded" while moral rules are "unconditionally obligatory, generalizable and impersonal insofar as they stem from concepts of welfare, justice, and rights" (56] p. 169-170).

Turiel and the other authors that have investigated this position (see e.g. [49] and [54] for reviews) point out that moral violations are perceived (both by children and adults) as more serious than the conventional ones; as independent from any authority that imposes them (like parents, teachers, governments or even God); and as ubiquitous, i.e. as not bound to any particular place, context, culture or habit. So, according to this characterization, while an act like e.g. eating with the hands is perceived as a conventional violation, which is not very serious and applies only in some places, contexts and cultures but not in others, and which depends on an authority, an act of violence is perceived as a serious moral violation, that applies everywhere and does not depend on any authority that imposes a restriction or compliance.

Nevertheless, the idea that it is possible to trace an univocal, transcultural and unanimous difference between conventional norms and moral norms on the basis of the criterion that the only properly moral violations are those related to welfare, justice, and rights has been challenged by a different research tradition, lead by Haidt. Haidt's research shows in fact that what people recognize as properly moral violations depends on both social and cultural factors.

As far as social factors are concerned, Haidt's experiments show that the moral intuitions of people are deeply influenced by their socioeconomic status, therefore, indirectly, by their level of education, and more generally, by the variety of their contacts and by the amount and quality of their experiences and knowledge (see e.g. 29]). According to Haidt, theories relying on Turiel's position, which narrow the moral domain to issues of harm/care and fairness/reciprocity/justice are 'parochial' and biased by the fact that the researchers carrying out these studies are more often liberal, and investigate therefore only the moral values they recognize as such. In addition, the data collected by these researchers are further biased by the fact that experimental subjects typically come from the same social group, since they are mostly university students and colleagues. Haidt shows that while well-educated people with a high socioeconomic status, especially secular and liberal Westerners, do actually identify the domain of morality only with phenomena related to welfare, justice, and rights, people of low socio-economic status consider as properly moral also other kinds of violations involving things which are offensive, disrespectful or disgusting (as e.g. having sex with a chicken carcass or cleaning the toilet with the National flag: see 29]). 
It is to explain these aspects that Haidt appeals to the cultural factors that influence moral intuitions. Further studies by Haidt carried out on cultures other than the ones usually considered in academic research - i.e. studies investigating cultures other than the North American and European ones, or also addressed to conservatives in Western cultures - show that people may also consider as properly moral (and not just as conventional) issues of in-group/loyalty, authority/respect and purity/sanctity (see e.g [25], 28] and [26]). As Haidt points out also relating his point to the empirical research of other authors : "in most cultures the social order is a moral order, and rules about clothing, gender roles, food, and forms of address are profoundly moral issues. [...] In many cultures the social order is a sacred order as well." (28 p. 371) And further: "[...] only an elite American college population limited the moral domain to matters of harm, rights, and justice. For other groups, particularly for low socioeconomic status groups in Brazil and in the United States, actions that were disrespectful or disgusting were said to be morally wrong (universally wrong and unchangeable) even when respondents specifically stated that nobody was harmed by the action." (28 p. 372)

If we cross Haidt's investigations with the conclusions reached by Turiel we achieve a result which is as unsurprising as it is interesting and difficult to explain on the basis of Haidt's theory. (a) Firstly, although not all cultures or groups restrict the domain of morality to issues related solely to harm/care, fairness/reciprocity/justice, these nevertheless represent something like a 'lowest common denominator' or 'hard core' of moral cognition, which is shared by everyone (i.e. by people of any origin, educated or not, liberal or conservative, religious or secular, belonging to one culture or to another). The idea of a lowest common denominator of moral sense focused on harm-fairness-based violations is also shared by other studies, as e.g. the ones that try to show that moral judgments may be explained by analogy with Chomsky's grammatically judgments (see e.g. [39] and [30]).

(b) Secondly the research by Haidt and his colleagues devoted specifically to the correlation between moral intuitions and low/high socio-economic status shows that a high level of education, wide variety of contacts, as well as having a large amount and high quality of experiences and knowledge (features which typically go with a high socio-economic status) strongly lead the individuals' moral intuitions to focus mostly or exclusively on this 'hard core' rather than on other aspects. This suggests that - when the individuals' level of education (in a wide sense) increases - they 'learn' to distinguish a moral and a conventional domain according to the criteria put forward by Turiel. That is to say that they learn to identify properly moral violations related exclusively to issues of welfare, justice, and rights and to distinguish them from other kinds of violations, concerning disgusting or disrespectful, but harmless actions. (see [29]).

In this sense, one could say that Haidt's studies show indirectly that a higher level of education (in a wide sense) acts as a 'natural antibody' against the tendency exhibited by people of a low socio-economic status to extend 
the domain of moral violations to harmless actions, which are nevertheless considered as disgusting or disrespectful. So, we could suggest that a high level of education (i.e. a high socio-economic status) rescues the moral positions of people from the dominance of the principles they take from their culture and establishes a transcultural connection or unity around the principle that morality has to do with harm and fairness only, while disrespectful or disgusting actions as well as contraventions of religious prescriptions or 'good manners' cannot be considered moral violations.

The fact that this principle seems to be present in all social groups corroborates the idea that questions related to harm-fairness are a necessary part of the human sense of morality. Still, the idea of morality developed by many cultures incorporates also other aspects like in-group/loyalty, authority/respect and purity/sanctity, whose respect is useful for the survival of the culture and of the social order within its borders. Nevertheless, these aspects are not a proper expression of an authentic moral sense, as this is represented by common sense.

As has been pointed out e.g. by social psychologists, loyalty to - and more generally favoritism towards - the in-group is typical in all intergroup relationships. Still, this phenomenon is not necessarily positive from a moral point of view. On the contrary, it sometimes leads to morally negative consequences: in fact, acting unfairly or violently toward people that do not belong to the in-group or, in the worst case scenario, racism towards or infra-humanizing the out-group are possible consequences of in-group loyalty. The same point also applies to respect for authority. To respect authority isn't always or necessarily morally positive: when the orders issued by an authority are not morally admissible, the truly moral reaction is refusing to follow them. And this presupposes once again that the capacity to think in a critical and autonomous way is required in order to act morally and that neither respect for authority nor loyalty towards the in-group are per se moral attitudes.

The case of purity/sanctity is similar, although not identical in the sense that it does not describe a social phenomenon, but rather virtues defined by a religious tradition. Each religious tradition has developed its own ideals of purity and sanctity and imposes these on its followers not only through teachings, but also by violently forcing people to embrace them. Historically, respect for ideals like those of purity and sanctity often required the personal sacrifice of sexuality, health or even life. Such sacrifices are perceived as morally positive only by the followers of the religion that imposes them and often only by the most fanatic ones, while persons external to the group or even members of the group who have distanced themselves from the most strict - one could say: inhumane - aspects of their religion consider their imposition as morally impermissible.

These characteristics of in-group/loyalty, authority/respect and purity/ sanctity are incompatible with the idea that the higher expression of morality is the capacity of a person to resist blindly following the rules of his/her group or culture, but to autonomously evaluate whether they are good or acceptable 
from a moral point of view. According to the principles of in-group/loyalty and authority/respect, for example, the dissident never acts in a morally right manner, since he/she is not loyal to his/her in-group and disrespects the authority within his/her group, i.e. the laws and the rules of his/her group. As far as purity/sanctity is concerned, not only it is highly culturally variable what specifically should be considered pure or saintly, but in many cultures or subgroups these notions have been completely dismissed. As with the case of in-group/loyalty and authority/respect it is easy to imagine the case of a dissident who fights against the idea of purity and sanctity defended by his/her group, whose actions are nevertheless considered unanimously highly moral by all persons external to the group, and even by his/her group later in time.

(a) These remarks give further support to the idea of a lowest common denominator of moral sense focused on harm-fairness-based violations, i.e. they bear out the thesis of a trans-group moral similarity, in contrast to Heidt's position that morality is highly culturally dependent. On the contrary, morality seems to be intrinsically connected with the capacity to think critically and autonomously from culturally transmitted principles. (b) Furthermore, our analysis points out that a higher level of education modifies people moral thinking and strongly influences the individuals' moral intuitions to focus mostly or exclusively on this common denominator rather than on other aspects connected mainly with cultural beliefs about how one should behave.

If we admit that a primary consequence of a higher level of education is that people's concepts become broader - in particular, that people learn that some beliefs are highly dependent on specific cultures and religions (i.e. on the 'enculturation' each of us is a victim of) - and that people learn and develop the habit of reasoning in a more explicit and critical manner, than these data confirm a continuist interpretation of moral judgment. In fact, they suggest that - even though people often have moral intuitions that comply with the principles of their culture - these are neither the only moral judgments people can reach nor the best possible moral judgments humans can aim for. When we are able to reason slowly and explicitly and to rely on better and more broadly defined concepts, our moral responses can potentially become more 'moral' in the sense that they can overcome cultural factors and limitations and give rise to a transculturally accepted moral stance that best expresses our common moral sense.

\section{Conclusion}

On the basis of our discussion we would like to conclude that a continuist interpretation of intuitionism and rationalism seems to be more plausible then a discontinuist one, since an inferentially and logically based information process offers - at least potentially - better theoretical instruments to explain 
moral judgments in the various and flexible forms they assume in different contexts and situations.

Within the continuist approach we subscribe to neither slow nor fast processing is assumed to be immune from errors; however, since intuitions work on the basis of information that is limited and filtered, they are not our best shot in order to form a truly moral stance: the moral stances formed on the basis of intuitions run the risk of not being moral at all. On the other hand, the continuist interpretation must not be mistaken for an abstract ideal of moral reasoning, according to which reasoning may rely on unlimited resources and information. Indeed, reasoning is meant as information processing working on logical and inferential relations that refer to the semantic properties of the information processed. So defined, reasoning turns out to be bounded by specific constraints since it can work only on the available information - i.e. on the concepts we have. Furthermore, according to the position we present here, to reason may not be the first and spontaneous reaction humans have when they face a new situation. In fact, it is often when people meet an impasse, a contradiction or a novel or surprising situation that the natural biases of the cognitive system may be overcome, abandoning the use of heuristics or stereotypes and incorporating more information.

The continuist interpretation challenges the hypothesis of moral intuitionism according to which moral intuitions are rigidly driven by culturally learned principles. We argue that the example of moral dissidence shows clearly that humans are equipped with a moral sense which can be independent from the moral principles recognized by one's own culture, group or subgroup. Furthermore, the analysis we propose suggests that since intuitions are the product of our "enculturation", they are heavily undermined by cultural biases and are, as such, not the best means we have to come to a truly moral judgment. One way to allow culture to play a positive rather than a restraining role is through education. Since a higher level of education contributes both to enriching our knowledge, thereby broadening our horizons cross-culturally, and developing the habit of reasoning in a more explicit and critical manner, we suggest that a higher level of education con potentially (i.e. given the proper motivation) increase our capacity to reason and to reach judgments free of prejudices and therefore better formulate a moral point of view. In this, we agree with Petty and Wegener [46] when they state that people with low capacities for high elaboration and/or low motivation tend to be easy victims of biasing effects caused by their prejudices, while conscious elaborative processes help to get rid of these biases.

\section{References}

1. Allport, G.W.: The Nature of Prejudice. Addison-Wesley, Reading (1954)

2. Banaji, M.R., Hardin, C.D.: Automatic Stereotyping. Psychological Science 7, 136-141 (1996) 
3. Bodenhausen, G.V., Macrae, C.N.: Stereotype Activation and Inhibition. In: Wyer, R. (ed.) Stereotype Activation and Inhibition, Erlbaum, Mahwah (1998)

4. Dellantonio, S., Job, R.: Morality According to a Cognitive Interpretation: A Semantic Model for Moral Behavior. In: Magnani, L., Carnielli, W., Pizzi, C. (eds.) Model-Based Reasoning in Science and Technology. SCI, vol. 314, pp. 495-517. Springer, Heidelberg (2010)

5. Devine, P.G.: Stereotypes and Prejudice: Their Automatic and Controlled Components. Psychological Science 56, 5-18 (1989)

6. Devine, P.G., Monteith, M.J.: Automaticity and Control in Stereotyping. In: Chaiken, S., Trope, Y. (eds.) Dual-Process Theories in Social Psychology. Guilfort, New York (1999)

7. Evans, J.S.B.T.: In Two Minds. Dual-Process Accounts of Reasoning. Trends in Cognitive Sciences 7, 454-459 (2003)

8. Evans, J.S.B.T.: How Many Dual-Process Theories Do We Need? One, Two, or Many? In: Evans, J.S.B.T., Frankish, K. (eds.) Two Minds. Dual Processes and Beyond, Oxford University Press, Oxford (2009)

9. Evans, J.S.B.T., Frankish, K.: In Two Minds: Dual Process and Beyond. Oxford University Press, Oxford (2009)

10. Evans, J.S.B.T., Over, D.E.: Rationality and Reasoning. Psychology Press, Hove (1996)

11. Fiske, S.T.: Examining the Role of Intent. Toward Understanding its Role in Stereotyping and Prejudice. In: Uleman, J.S., Bargh, J.A. (eds.) Unintended Thought. Guilfort, New York (1989)

12. Fiske, S.T., Taylor, S.E.: Social Cognition. McGraw-Hill, New York (1991)

13. Fodor, J.A.: The Modularity of Mind. An Essay on Faculty Psychology. MIT Press, Cambridge (1983)

14. Fodor, J.A.: Psychosemantics. The Problem of Meaning in the Philosophy of Mind. MIT Press, Cambridge (1987)

15. Fodor, J.A.: Concepts. Where the Cognitive Science Went Wrong. Clarendon Press, Oxford (1998)

16. Frankish, K.: Systems and Levels: Dual-system Theories and the PersonalSubpersonal Distinction. In: Evans, B.T., Frankish, K. (eds.) Two Minds. Dual Processes and Beyond. Oxford University Press, Oxford (2009)

17. Gigerenzer, G.: Gut Feelings: The Intelligence of the Unconscious. Penguin, London (2007)

18. Gigerenzer, G.: Moral Intuition = Fast and Frugal Heuristics? In: SinnottArmstrong, W. (ed.) Moral Psychology. The Cognitive Science of Morality: Intuition and Diversity, vol. 2. MIT Press, Cambridge (2008)

19. Gigerenzer, G., Todd, P.M.: The ABC Research Group: Simple Heuristics That Makes Us Smart. Oxford University Press, Oxford (1999)

20. Goldstein, D.G., Gigerenzer, G.: Fast and Frugal Forecasting. Journal of Forecasting 25, 760-772 (2009)

21. Habermas, J.: The Theory of Communicative Action: Reason and the Rationalization of Society, vol. 1. Beacon Press, Boston (1985)

22. Haidt, J.: The Emotional Dog and its Rational Tail: A Social Intuitionist Approach to Moral Judgment. Psychological Review 108, 814-834 (2001)

23. Haidt, J.: The Moral Emotions. In: Davidson, R.J., Scherer, K.R., Goldsmith, H.H. (eds.) Handbook of Affective Sciences. Oxford University Press, Oxford (2003) 
24. Haidt, J., Bjorklund, F.: Social Intuitionists Answer Six Questions About Moral Psychology. In: Sinnott-Armstrong, W. (ed.) Moral Psychology. The Cognitive Science of Morality: Intuition and Diversity, vol. 2. MIT Press, Cambridge (2008)

25. Haidt, J., Graham, J.: When Morality Opposes Justice: Conservatives Have Moral Intuitions That Liberals May Not Recognize. Social Justice Research 20, 98-116 (2007)

26. Haidt, J., Graham, J.: Planet of the Durkheimians, Where Community, Authority, and Sacredness are Foundations of Morality. In: Jost, J., Kay, H.T.A.C. (eds.) Social and Psychological Bases of Ideology and System Justification, Oxford University Press, Oxford (2009)

27. Haidt, J., Joseph, C.: Intuitive Ethics: How Innately Prepared Intuitions Generate Culturally Variable Virtues. Daedalus 133, 55-66 (2004)

28. Haidt, J., Joseph, C.: The Moral Mind: How Five Sets of Innate Intuitions Guide the Development of Many Cultural-Specific Virtues, and Perhaps Even Modules. In: Carruthers, P., Laurence, S., Stich, S. (eds.) The Innate Mind. Foundations and the Future, vol. 3, Oxford University Press, Oxford (2007)

29. Haidt, J., Koller, S., Diaz, M.: Affect, Culture and Morality, or it is Wrong to Eat Your Dog? Journal of Personality and Social Psychology 65, 612-628 (1993)

30. Hauser, M.D.: Moral Minds. How Nature Designed Our Moral Sense of Right and Wrong. Collins Publisher, New York (2006)

31. Kahneman, D., Slovic, P., Tversky, A.: Judgment Under Uncertainty: Heuristics and Biases. Cambridge University Press, Cambridge (1982)

32. Kahneman, D., Tversky, A.: Choices, Values, and Frames. Cambridge University Press, Cambridge (1982)

33. Kant, I.: The Critique of Practical Reason. Cambridge University Press, Cambridge (1788-1977)

34. van Knippenberg, D., van Knippenberg, A.: Social Categorization, Focus of Attention and Judgments of Group Opinions. British Journal of Social Psychology 33, 477-489 (1994)

35. Kohlberg, L.: Stage and Sequence. The Cognitive-Developmental Approach to Socialization. In: Goslin, D.A. (ed.) Handbook of Socialization Theory and Research, Rand McNally, Chicago (1969)

36. Macrae, C.N., Hewstone, M., Griffiths, R.J.: Processing Load and Memory for Stereotype-Based Information? European Journal of Social Psychology 23, 7787 (1993)

37. Macrae, C.N., Milne, A.B., Hewstone, Bodenhausen, G.V.: Stereotypes as Energy-Saving Devices: A Peek Inside the Cognitive Toolbox. Journal of Personality and Social Psychology 66, 37-47 (1994)

38. Magnani, L.: Abduction, Reason, and Science. Processes of Discovery and Explanation. Kluwer Academic, New York (2001)

39. Mikheil, J.: Rawls Linguistic Analogy. Ph.D. Thesis Cornell University Press, New York (2001)

40. Nervaez, D.: The Social Intuitionist Model: Some Counter-Intuitions. In: Sinnott-Armstrong, W. (ed.) Moral Psychology. The Evolution of Morality: Adaptations and Innateness, vol. 1. MIT Press, Cambridge (2008)

41. Nisbett, R.E.: The Geography of Thought. How Asians and Westerns Think Differently... and Why. Free Press, New York (2003) 
42. Nisbett, R.E., Cohen, D.: Culture of Honor: The Psychology of Violence in the South. Westview Press, Boulder (1996)

43. Pastore, L.: Intuition. In: Sandkühler, H.J. (ed.) Enzyklopädie Philosophie. Felix Meiner Verlag, Hamburg (2010)

44. Peirce, C.S.: Collected Papers. 1903 Harvard Lectures on Pragmatism. In: Hartshorne, C., Weiss, P.(ed.) vol. 5 (vols. I-VI). Harvard University Press, Cambridge (1931-1958)

45. Petty, R.E., Cacioppo, J.T.: The Elaboration Likelihood Model of Persuasion. In: Berkowitz, L. (ed.) Advances in Experimental Social Psychology, vol. 19. Academic Press, Orlando (1986)

46. Petty, R.E., Wegener, D.T.: The Elaboration Likelihood Model: Current Status and Controversies. In: Chaiken, S., Trope, Y. (eds.) Dual Process Theories in Social Psychology. Guilford Press, New York (1999)

47. Piaget, J.: The Moral Judgment of the Child. Free Press, New York (1932-1965)

48. Sloman, S.A.: The Empirical Case for Two Systems of Reasoning. Psychological Bulletin 119, 3-22 (1996)

49. Smetana, J.: Understanding of Social Rules. In: Bennett, M. (ed.) The Development of Social Cognition: The Child as Psychologist, Guilfort Press, New York (1993)

50. Smith, E.E.: Concepts and Categorization. In: Smith, E.E., Osherson, N.D. (eds.) Thinking. An Invitation to Cognitive Science, vol. 3. MIT Press, Cambridge (1995)

51. Smith, E.R., DeCoster, J.: Dual-Process Models in Social and Cognitive Psychology: Conceptual Integration and Links to Underlying Memory Systems. Personality and Social Psychology Review 4, 108-131 (2000)

52. Stangor, C.: The Study of Stereotyping, Prejudice, and Discrimination Within Social Psychology. A Quick History of Theory and Research. In: Nelson, T.D. (ed.) Handbook of Prejudice, Stereotyping and Discrimination. Psychology Press, New York (2009)

53. Stanovich, K.E.: Who Is Rational? Studies of Individual Differences in Reasoning. Lawrence Erlbaum Associates, Mahwah (2009)

54. Tisak, M.: Domains of Social Reasoning and Beyond. In: Vasta, R. (ed.) Annals of Child Development, vol. 11. Jessica Kingsley, London (1995)

55. Turiel, E.: The Culture of Morality. Social Development, Context and Conflict. Cambridge University Press, Cambridge (2002)

56. Turiel, E., Killen, M., Helwig, C.: Morality: Its Structure, Functions, and Vagaries. In: Kagan, J., Lamb, S. (eds.) The Emergence of Morality in Young Children. University of Chicago Press, Chicago (1987)

57. Uleman, J.S., Bargh, J.A.: Unintended Thought. Guilford, New York (1989)

58. Walton, D.: Abductive Reasoning. The University of Alabama Press, Tuscaloosa (2004) 